\title{
Distant Intracranial Metastasis
}

National Cancer Institute

\section{Source}

National Cancer Institute. Distant Intracranial Metastasis. NCI Thesaurus. Code C84360.

The presence of brain metastasis that originates from a cancer growth in a distant anatomic site. 